\title{
ON THE HALPHEN TRANSFORM OF ALGEBRAIC SPACE CURVES
}

\author{
ALFREDERIC JOSSE AND FRANÇOISE PÈNE
}

\begin{abstract}
The Halphen transform of a plane curve is the curve obtained by intersecting the tangent lines of the curve with the corresponding polar lines with respect to some conic. This transform has been introduced by Halphen as a branch desingularization method in [4] and has also been studied in 1, 7. We extend this notion to Halphen transform of a space curve and study several of its properties (birationality, degree, rank, class, desingularization).
\end{abstract}

\section{INTRODUCTION}

In [4, Halphen studied plane curve transformations based on a simple geometric construction. Given a plane curve $\mathcal{C}$, choosing a conic $\mathcal{K}$ in the same plane, every nonsingular point $m$ of $\mathcal{C}$ is mapped on the intersection of the tangent line $\mathcal{T}_{m} \mathcal{C}$ to $\mathcal{C}$ at $m$ with the polar of $m$ with respect to $\mathcal{K}$. The Halphen transform of $\mathcal{C}$ with respect to $\mathcal{K}$ is the Zariski closure of the image of $\mathcal{C}$ by this transformation. It is clear that this transformation separates branches at any multiple point of $\mathcal{C}$ with distinct tangents. Even more, in [4, Halphen shew namely that iterations of these transformations provide a branch desingularization process. In [1], Coolidge mentionned some properties of Halphen transforms. Further properties of these transforms have been carefully studied by Josse in [7.

In the present work, we extend the construction of Halphen transforms to curves of the three dimensional complex projective space $\mathbb{P}^{3}$. We show that these transformations can be used for desingularization. We study the birationality of the Halphen transformation. We establish also formulas for the degree, the rank and the class of Halphen transforms. The study of these space Halphen transforms is much more complicated than the original one for several reasons: these transforms don't act on a hypersurface, all space curves are not complete intersection of two hypersurfaces, the local parametrization of their branches is more complicated than for space curves (for which it is simply given by a single Puiseux expansion [5, 11]), etc.

Let $\mathbb{P}^{3}:=\mathbb{P}(\mathbf{W})$ where $\mathbf{W}$ is a four dimensional complex vector space. Let $\mathcal{C}$ be an algebraic curve of $\mathbb{P}^{3}$ and $\mathcal{Q}$ be an irreducible quadric of $\mathbb{P}^{3}$. For $m \in \mathcal{C}$, we write $\Phi_{\mathcal{C}, \mathcal{Q}}(m)$ for the intersection point of the tangent line $\mathcal{T}_{m} \mathcal{C}$ with the polar plane $\delta_{m} \mathcal{Q}$ of $\mathcal{Q}$ with respect to $m \in \mathcal{C}$ (when this point is well defined). The Halphen transform $\mathcal{C}^{\mathcal{Q}}$ of $\mathcal{C}$ with respect to $\mathcal{Q}$ is the Zariski closure of the image of $\mathcal{C}$ by $\Phi_{\mathcal{C}, \mathcal{Q}}$. As for plane curves, by definition, this transformation separates branches of nodes of $\mathcal{C}$. Recall that the degree of $\mathcal{C}$ corresponds to the number of intersection points of $\mathcal{C}$ with a generic plane of $\mathbb{P}^{3}$, that its rank corresponds to the number of its tangent lines intersecting a generic line of $\mathbb{P}^{3}$ and that its class is the number of its osculating planes passing through a generic point of $\mathbb{P}^{3}$. We also write $g(\mathcal{C})$ for the (geometric) genus of $\mathcal{C}$.

Theorem 1 (Numerical characters of Halphen transform). Let $\mathcal{C} \subset \mathbb{P}^{3}$ be an irreducible curve. For a generic for a generic quadric $\mathcal{Q} \subset \mathbb{P}^{3}$, the Halphen map $\Phi_{\mathcal{C}, \mathcal{Q}}$ is birational, so the Halphen

Date: May 9, 2018.

2000 Mathematics Subject Classification. 14J99,14H50,14E05,14N05,14N10.

Key words and phrases. Halphen's tranform, space curve, desingularization, degree. 
transform preserves the genus, i.e.

$$
g\left(\mathcal{C}^{\mathcal{Q}}\right)=g(\mathcal{C})
$$

Moreover, for a generic quadric $\mathcal{Q} \subset \mathbb{P}^{3}$, the degree, the rank and the class of $\mathcal{C}^{\mathcal{Q}}$ are given by the following formulas

$$
\begin{gathered}
\operatorname{deg} \mathcal{C}^{\mathcal{Q}}=\operatorname{deg} \mathcal{C}+\operatorname{rank} \mathcal{C} \\
\operatorname{rank} \mathcal{C}^{\mathcal{Q}}=2(\operatorname{deg} \mathcal{C}+\operatorname{rank} \mathcal{C}+g(\mathcal{C})-1)-k_{0}\left(\mathcal{C}^{\mathcal{Q}}\right)
\end{gathered}
$$

and

$$
\operatorname{class} \mathcal{C}^{\mathcal{Q}}=3 \operatorname{deg} \mathcal{C}+3 \operatorname{rank} \mathcal{C}+6 g(\mathcal{C})-6-2 k_{0}\left(\mathcal{C}^{\mathcal{Q}}\right)-k_{1}\left(\mathcal{C}^{\mathcal{Q}}\right),
$$

where $k_{i}\left(\mathcal{C}^{\mathcal{Q}}\right)$ is the $i$-th stationary index of $\mathcal{C}^{\mathcal{Q}}$.

Let us indicate that (3) and (4) follow directly from (11) and (22) thanks to formulas established by Piene in [9] and recalled in Section 6.1. Let us precise that $k_{0}(\mathcal{C})$ corresponds to the number of cusps of $\mathcal{C}$ (with their multiplicities) and that $k_{1}(\mathcal{C})$ corresponds to the number of inflection points of $\mathcal{C}$ (with their multiplicities). The precise definitions of these quantities are recalled in Section 6.1

Remark 2. Let $\mathcal{C}$ be an irreducible algebraic curve of $\mathbb{P}^{3}$.

Proposition 15 below gives the type of the branch $\Phi_{\mathcal{C}, \mathcal{Q}}(\mathcal{B})$ of $\mathcal{C}^{\mathcal{Q}}$ for any type of branch $\mathcal{B}$ of $\mathcal{C}$ for a generic quadric $\mathcal{Q} \subset \mathbb{P}^{3}$. This precise result has several consequences.

First, it enables the computation of the indices $k_{0}\left(\mathcal{C}^{\mathcal{Q}}\right)$ and $k_{1}\left(\mathcal{C}^{\mathcal{Q}}\right)$ for a generic quadric $\mathcal{Q} \subset \mathbb{P}^{3}$, given the type of the singular branches and of the smooth inflectional branches of $\mathcal{C}$.

Second, it ensures that the Halphen transform (for a generic quadric $\mathcal{Q}$ ) decreases the order of contact of non singular inflectional branches of $\mathcal{C}$ with their tangent lines and that, except in very special cases, it also decreases the singularities of the singular branches of $\mathcal{C}$ (see Corollary 16).

In Section 2, we recall some facts on tangent curves, polar surfaces, rank and tangent developable. In Section 3, we detail the construction of the Halphen transform of a space curve. In Section 4, we prove the degree formula. In Section 5, we see that the Halphen transform can be used as a branch desingularization method. In Section 6 we prove the rank and class formulas and illustrate them on examples. In Section 7 we prove the birationality of the Halphen map $\Phi_{\mathcal{C}, \mathcal{Q}}$ for a generic quadric $\mathcal{Q}$.

\section{RECALLS ON TANGENCY AND RANK}

Let us recall that the tangent plane $\mathcal{T}_{m} \mathcal{S}$ to an algebraic surface $\mathcal{S}=V(\chi) \subset \mathbb{P}^{3}$ (with $\left.\chi \in \operatorname{Sym}_{d} \mathbf{W}^{\vee}\right)$ at a nonsingular 1 point $m$ of $\mathcal{S}$ is the plane $\mathcal{T}_{m} \mathcal{S}=V(d \chi(\mathbf{m})) \subset \mathbb{P}^{3}$, where $\mathbf{m} \in \mathbf{W} \backslash\{\mathbf{0}\}$ is a representant of $m \in \mathbb{P}^{3}$ and where $d \chi(\mathbf{m}) \in \mathbf{W}^{\vee}$ is the differential of $\chi$ at $\mathbf{m}$.

Analogously, given an algebraic curve $\mathcal{C}=\bigcap_{i=1}^{I} \mathcal{S}_{i}$, where $\mathcal{S}_{i}=V\left(\chi^{(i)}\right) \subset \mathbb{P}^{3}$ are surfaces (with $I \geq 2$ and $\chi^{(i)} \in \operatorname{Sym}_{d_{i}} \mathbf{W}^{\vee}$ ), the tangent line $\mathcal{T}_{m} \mathcal{C}$ to $\mathcal{C}$ at a nonsingular ${ }^{2}$ point $m$ of $\mathcal{C}$ is the line $\mathcal{T}_{m} \mathcal{C}=\bigcap_{i=1}^{I} V\left(d \chi^{(i)}(\mathbf{m})\right) \subset \mathbb{P}^{3}$.

In practice, we will use projective coordinates (by fixing a vector basis $\left(\mathbf{e}_{1}, \mathbf{e}_{2}, \mathbf{e}_{3}, \mathbf{e}_{4}\right)$ of $\mathbf{W}$ ). We represent each point $m$ of $\mathbb{P}^{3}$ by its coordinates $[x: y: z: t]$ and we write $\mathbf{m}=(x, y, z, t) \in$ $\mathbf{W} \backslash\{\mathbf{0}\}$. In coordinates, we identify $\chi \in S y m_{d} \mathbf{W}^{\vee}$ with an homogeneous polynomial $F \in$ $\mathbb{C}[x, y, z, t]$ of degree $d$ and we write as usual $F_{x}, F_{y}, F_{z}$ and $F_{t}$ for its partial derivatives.

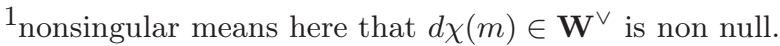

2 nonsingular means here that $\left.\operatorname{Vect}\left(d \chi^{(1)}(m), \ldots, d \chi^{(I)}\right)(m)\right) \subset \mathbf{W}^{\vee}$ has dimension 2.
} 
We also identify $d \chi(\mathbf{m})$ with the gradient $\nabla F(\mathbf{m})=\left[F_{x}(\mathbf{m}): F_{y}(\mathbf{m}): F_{z}(\mathbf{m}): F_{t}(\mathbf{m})\right]$. Let $\mathcal{H}^{\infty}:=V(t)$ be the plane at infinity.

2.1. Tangent curve. We write $\mathbb{G}(1,3)$ for the set of projective lines of $\mathbb{P}^{3}$. As usual we write $\mathbb{T C} \subset \mathbb{G}(1,3)$ for the tangent curve of $\mathcal{C}$, that is the Zariski closure of the set of tangent lines to $\mathcal{C}$ (see for example [6, p. 188]). We recall that $\mathbb{G}(1,3)$ is embedded in $\mathbb{P}^{5}$ via the Plücker embedding.

Up to a linear change of variables, we assume that $\mathcal{C} \not \subset \mathcal{H}^{\infty}$. If $\mathcal{C}$ is contained in a curve $\mathcal{C}_{1}=V(F, G) \subset \mathbb{P}^{3}$ with $F, G \in \mathbb{C}[x, y, z, t]$ homogeneous. Then, for any $m \in \mathcal{C} \backslash\left(\operatorname{Sing}\left(\mathcal{C}_{1}\right) \cup \mathcal{H}^{\infty}\right)$, the tangent line $\mathcal{T}_{m} \mathcal{C}=\mathcal{T}_{m} \mathcal{C}_{1}$ is the line of $\mathbb{P}^{3}$ passing through the points $m$ and $\mathfrak{t}_{\infty, \mathcal{C}_{1}}(m) \in \mathbb{P}^{3}$, where $\mathfrak{t}_{\infty, \mathcal{C}_{1}}(m)$ is the point of $\mathbb{P}^{3}$ with coordinates

$$
\mathfrak{t}_{\infty, \mathcal{C}_{1}}(\mathbf{m}):=\left(\begin{array}{c}
F_{y}(\mathbf{m}) G_{z}(\mathbf{m})-F_{z}(\mathbf{m}) G_{y}(\mathbf{m}) \\
F_{z}(\mathbf{m}) G_{x}(\mathbf{m})-F_{x}(\mathbf{m}) G_{z}(\mathbf{m}) \\
F_{x}(\mathbf{m}) G_{y}(\mathbf{m})-F_{y}(\mathbf{m}) G_{x}(\mathbf{m}) \\
0
\end{array}\right) \in \mathbb{C}^{4} .
$$

Via the Plücker embedding [3], $\mathcal{T}_{m} \mathcal{C}$ is identified with $\lambda_{\mathcal{C}_{1}}(m)$ with $\lambda_{\mathcal{C}_{1}}: \mathbb{P}^{3} \rightarrow \mathbb{P}(\stackrel{2}{\wedge} \mathbf{W}) \cong \mathbb{P}^{5}$ given on coordinates by 3

$$
\boldsymbol{\lambda}_{\mathcal{C}_{1}}(\mathbf{m})=\left(\bigwedge^{2}\left(\mathbf{m} \mathfrak{t}_{\infty, \mathcal{C}_{1}}(\mathbf{m})\right)\right) \in \mathbb{C}^{6}
$$

and so $\mathbb{T C}$ is identified to $\overline{\lambda_{\mathcal{C}_{1}}(\mathcal{C})}$. Due to the Euler Formula applied to $F$ and $G$, on $\mathcal{C}$, we have

$$
\lambda_{\mathcal{C}_{1}}=-t\left(\begin{array}{c}
F_{z} G_{t}-G_{z} F_{t} \\
F_{t} G_{y}-F_{y} G_{t} \\
F_{y} G_{z}-F_{z} G_{y} \\
F_{x} G_{t}-G_{x} F_{t} \\
F_{z} G_{x}-F_{x} G_{z} \\
F_{x} G_{y}-F_{y} G_{x}
\end{array}\right)
$$

2.2. Dual tangent curve. We write $\mathbb{G}\left(1, \mathbb{P}\left(\mathbf{W}^{\vee}\right)\right)$ for the set of projective lines of $\mathbb{P}\left(\mathbf{W}^{\vee}\right)$. We consider the duality between $\mathbb{G}(1,3)$ and $\mathbb{G}\left(1, \mathbb{P}\left(\mathbf{W}^{\vee}\right)\right)$ which, to $\mathcal{L} \in \mathbb{G}(1,3)$, associates $\mathcal{L}^{*}=\left\{\varphi \in \mathbb{P}\left(\mathbf{W}^{\vee}\right): \quad \mathcal{L} \subset V(\varphi) \subset \mathbb{P}^{3}\right\}$. It is then natural to consider the dual tangent curve $(\mathbb{T C})^{*}=\left\{\mathcal{L}^{*}: \mathcal{L} \in \mathbb{T C}\right\} \subset \mathbb{G}\left(1, \mathbb{P}\left(\mathbf{W}^{\vee}\right)\right)$. Observe that $(\mathbb{T C})^{*}$ is the Zariski closure of $\left\{\left(\mathcal{T}_{m} \mathcal{C}\right)^{*}, m \in \mathcal{C} \backslash \operatorname{Sing}(\mathcal{C})\right\}$ and that $\left(\mathcal{T}_{m} \mathcal{C}\right)^{*}$ corresponds to the set of projective planes of $\mathbb{P}^{3}$ containing $\mathcal{T}_{m} \mathcal{C}$. Via the Plücker embedding, $(\mathbb{T C})^{*}$ is identified with the Zariski closure of the image of $\mathcal{C}$ by the rational map $\vartheta_{\mathcal{C}_{1}}: \mathbb{P}^{3} \rightarrow \mathbb{P}\left(\bigwedge^{2} \mathbf{W}^{\vee}\right) \cong \mathbb{P}^{5}$ given on coordinates by

$$
\boldsymbol{\vartheta}_{\mathcal{C}_{1}}(\mathbf{m})=\bigwedge^{2}(\nabla F(\mathbf{m}) \nabla G(\mathbf{m})) \in \mathbb{C}^{6}
$$

Comparing $\vartheta_{\mathcal{C}_{1}}$ with (5), we conclude that $\mathbb{T C}$ and $(\mathbb{T C})^{*}$ are identified via Plücker embeddings up to a linear change of coordinates. In particular, their images via Plücker embeddings in $\mathbb{P}^{5}$ have same degree. This explains why $\mathbb{T C}$ and $(\mathbb{T C})^{*}$ are often considered as the same object.

${ }^{3}$ we recall that where, in coordinates, $\stackrel{2}{\wedge}(\mathbf{u} \mathbf{v})=\left(\begin{array}{l}u_{1} v_{2}-u_{2} v_{1} \\ u_{1} v_{3}-u_{3} v_{1} \\ u_{1} v_{4}-u_{4} v_{1} \\ u_{2} v_{3}-u_{3} v_{2} \\ u_{2} v_{4}-u_{4} v_{2} \\ u_{3} v_{4}-u_{4} v_{3}\end{array}\right) \in \mathbb{C}^{6}$ for any $\mathbf{u}, \mathbf{v} \in \mathbf{W}$ 
2.3. Polar surface. We call polar surface from $\beta \in\left(\stackrel{2}{\wedge} \mathbf{W}^{\vee}\right)^{\vee} \cong \mathbb{C}^{6}$ of the complete intersection curve $\mathcal{C}_{1}$ the surface $V\left(\beta \circ \boldsymbol{\vartheta}_{\mathcal{C}_{1}}\right) \subset \mathbb{P}^{3}$. This extends to the three dimensional case the notion of polar curves of plane projective curves.

Lemma 3. Let $m$ be a non singular point of $\mathcal{C}_{1}$. Let $\ell \subset \mathbb{P}^{3}$ be a projective line containing $a, b \in \mathbb{P}^{3}$ (with $\left.a \neq b\right)$, then $\mathcal{T}_{m} \mathcal{C}$ intersects the line $\ell$ if and only if

$$
\left\langle\bigwedge^{2}(\nabla F(\mathbf{m}) \nabla G(\mathbf{m})), \bigwedge^{2}(a b)\right\rangle=0
$$

in coordinates 4

Proof. The fact that $\mathcal{T}_{m} \mathcal{C}$ intersects the line $\ell$ is equivalent to the existence of $(u, v) \in \mathbb{C}^{2} \backslash(0,0)$ such that $\langle\nabla F(\mathbf{m}), u a+b v\rangle=0$ and $\langle\nabla G(\mathbf{m}), u a+b v\rangle=0$, i.e. $\operatorname{det}\left(\begin{array}{cc}\langle\nabla F(\mathbf{m}), a\rangle & \langle\nabla F(\mathbf{m}), b\rangle \\ \langle\nabla G(\mathbf{m}), a\rangle & \langle\nabla G(\mathbf{m}), b\rangle\end{array}\right)=$ 0 , which leads to (6).

Observe that the set of $m \in \mathbb{P}^{3}$ satisfying (6) corresponds to the polar surfaces from $\beta$ corresponding to the Plücker embedding of $\ell$.

2.4. Link with the rank. The rank of $\mathcal{C}$ is usually described as the degree of the tangent developable surface of $\mathcal{C}$, i.e. the Zariski closure of the union of the tangent curves of $\mathcal{C}$. We have also the following interpretation of the rank of $\mathcal{C}$ in terms of the tangent curve.

Lemma 4. The rank of $\mathcal{C}$ corresponds to the degree in $\mathbb{P}^{5}$ of the Plücker embedding of $\mathbb{T C}$.

Proof. We work with coordinates. Recall that the image by the Plücker embedding of $\mathbb{G}(1,3)$ or $\mathbb{G}\left(1, \mathbb{P}\left(\mathbf{W}^{\vee}\right)\right)$ is $\mathcal{K}=V\left(x_{1} x_{6}-x_{2} x_{5}+x_{3} x_{4}\right) \subset \mathbb{P}^{5}$ if we write $\left[x_{1}: \ldots: x_{6}\right]$ for the coordinates of a point of $\mathbb{P}^{5}$. Due to Lemma 3 (and the remark following this lemma), it is enough to prove that for a generic $A=\left[a_{6}:-a_{5}: a_{4}: a_{3}:-a_{2}: a_{1}\right] \in \mathcal{K}$, the hyperplane $\mathcal{H}_{A}=V(\langle A, \cdot\rangle) \subset \mathbb{P}^{5}$ intersects $\tilde{\mathcal{C}}:=\overline{\vartheta_{\mathcal{C}_{1}}(\mathcal{C})} \subset \mathbb{P}^{5}$ transversally. Indeed, due to the Bezout theorem, this will imply that

$$
\#\left(\ell_{A} \cap \mathcal{C}\right)=\#\left(\mathcal{H}_{A} \cap \overline{\vartheta_{\mathcal{C}_{1}}(\mathcal{C})}\right)=\operatorname{deg} \overline{\vartheta_{\mathcal{C}_{1}}(\mathcal{C})}
$$

where $\ell_{A}$ is the projective line of $\mathbb{P}^{3}$ corresponding to $A \in \mathcal{K}$ via Plücker embedding.

Observe that this is not obvious since the hyperplane $\mathcal{H}_{A}$ with $A \in \mathcal{K}$ corresponds to the tangent hyperplane to $\mathcal{K}$ at $\left[a_{1}: a_{2}: a_{3}: a_{4}: a_{5}: a_{6}\right]$ and so these hyperplanes are very particular.

We assume that $\mathcal{H}_{A} \cap \tilde{\mathcal{C}} \subset \vartheta_{\mathcal{C}_{1}}(\mathcal{C})$. This is true for a generic $A \in \mathcal{K}$ since the set of projective hyperplanes intersecting the finite set of points $\tilde{\mathcal{C}} \backslash \vartheta_{\mathcal{C}_{1}}(\mathcal{C})$ has codimension 2 in the projective space of projective hyperplanes of $\mathbb{P}^{5}$.

Now let us prove that $\mathcal{H}_{A}$ intersects $\tilde{\mathcal{C}}$ transversally in the open set $\left\{x_{i} \neq 0\right\}$ for every $i=$ $1, \ldots, 6$. Take for example $i=6$ and assume $a_{6} \neq 0$. We set $a_{6}=1$ to simplify. In the chart $x_{6}=1$, $\tilde{C}=\Phi\left(\mathcal{C}^{(0)}\right)$ and $\mathcal{H}_{A} \cap \mathcal{K}=\Phi\left(\mathcal{H}_{\left(a_{2}, a_{3}, a_{4}, a_{5}\right)}^{(0)}\right)$ where $\Phi\left(x_{2}, x_{3}, x_{4}, x_{5}\right)=\left[x_{2} x_{5}-x_{3} x_{4}: x_{3}: x_{4}: x_{5}\right]$, where $\mathcal{C}^{(0)} \subset \mathbb{C}^{4}$ corresponds to the image of $\mathcal{C}$ by the projection $\left[x_{1}: x_{2}: x_{3}: x_{4}: x_{5}: 1\right] \mapsto$ $\left(x_{2}, x_{3}, x_{4}, x_{5}\right)$ and where

$$
\mathcal{H}_{\left(a_{2}, a_{3}, a_{4}, a_{5}\right)}^{(0)}=V\left(\left(x_{2}-a_{2}\right)\left(x_{5}-a_{5}\right)-\left(x_{3}-a_{3}\right)\left(x_{4}-a_{4}\right)\right) \subset \mathbb{C}^{4} .
$$

\footnotetext{
${ }^{4}$ with the classical notation $\langle A, B\rangle=\sum_{i=1}^{6} a_{i} b_{i}$ for any $A=\left(a_{1}, \ldots, a_{6}\right)$ and $B=\left(b_{1}, \ldots, b_{6}\right)$ in $\mathbb{C}^{6}$.
} 
Indeed $\mathcal{H}_{A} \cap \mathcal{K}=V\left(x_{1}-a_{5} x_{2}+a_{4} x_{3}+a_{3} x_{4}-a_{2} x_{5}+a_{1} x_{6}, x_{1} x_{6}-x_{2} x_{3}+x_{4} x_{5}\right)$ and so $\mathcal{H}_{A} \cap \mathcal{K}=$ $\Phi\left(\mathcal{H}_{A}^{(0)}\right)$ with

$$
\mathcal{H}_{A}^{(0)}=V\left(a_{5} x_{2}-a_{4} x_{3}-a_{3} x_{4}+a_{2} x_{5}-a_{1}-\left(x_{2} x_{3}-x_{4} x_{5}\right) \subset \mathbb{C}^{4},\right.
$$

but this corresponds exactly to $\mathcal{H}_{\left(a_{2}, a_{3}, a_{4}, a_{5}\right)}^{(0)}$ (since $\left.a_{1}=a_{2} a_{5}-a_{3} a_{4}\right)$. Now noticing that $\mathcal{C}^{(0)}$ is a curve of $\mathbb{C}^{4}$ and that $\mathcal{H}_{\left(a_{2}, a_{3}, a_{4}, a_{5}\right)}^{(0)}=\left(a_{2}, a_{3}, a_{4}, a_{5}\right)+\mathcal{H}_{(0,0,0,0)}^{(0)}$. We conclude that, for a generic $A \in \mathcal{K}, \mathcal{C}^{(0)}$ and $\mathcal{H}_{\left(a_{2}, a_{3}, a_{4}, a_{5}\right)}^{(0)}$ have transverse intersection and so $\tilde{\mathcal{C}}$ and $\mathcal{H}_{A}$ have also transverse intersection outside $V\left(x_{6}\right)$ (since the differential $D \Phi\left(x_{2}, x_{3}, x_{4}, x_{5}\right)$ is injective).

\section{HALPHEN TRANSFORM}

3.1. Definition. We recall that the polar $\delta_{m_{1}}(\mathcal{Q})$ of an irreducible quadric $\mathcal{Q}=V(Q) \subset \mathbb{P}^{3}$ with respect to a point $m_{1} \in \mathbb{P}^{3}$ is the projective plane $V\left(\Delta_{\mathbf{m}_{1}} Q\right) \subset \mathbb{P}^{3}$, with the usual notation $\Delta_{\mathbf{m}_{1}} Q=x_{1} Q_{x}+y_{1} Q_{y}+z_{1} Q_{z}+t_{1} Q_{t}$ if $\mathbf{m}_{1}=\left(x_{1}, y_{1}, z_{1}, t_{1}\right) \in \mathbf{W}$. Observe that, given $Q$ as above, for a generic $m_{1} \in \mathbb{P}^{3}, \delta_{m_{1}}(\mathcal{Q})$ is the projective plane passing through the points of $\mathcal{Q}$ at which the tangent plane to $\mathcal{Q}$ contains $m_{1}$.

Definition 5. Let $\mathcal{Q}=V(Q) \subset \mathbb{P}^{3}$ be an irreducible quadric and $\mathcal{C} \subset \mathbb{P}^{3}$ be an irreducible curve. The Halphen transform $\mathcal{C}^{\mathcal{Q}}$ of $\mathcal{C}$ with respect to $\mathcal{Q}$ is the Zariski closure of the set of intersection points $\Phi_{\mathcal{C}, \mathcal{Q}}(m)$ of the tangent line $\mathcal{T}_{m} \mathcal{C}$ to $\mathcal{C}$ at $m$ with the polar $\delta_{m} \mathcal{Q}$ of $\mathcal{Q}$ with respect to $m$, when $m$ varies on $\mathcal{C}$.

With this definition, $\Phi_{\mathcal{C}, \mathcal{Q}}(m)$ is the unique conjugated point of $m$, with respect to $\mathcal{Q}$, belonging to $\mathcal{T}_{m} \mathcal{C}$. Let $\mathcal{C}_{1}=V(F, G) \subset \mathbb{P}^{3}$ be a complete intersection curve containing $\mathcal{C}$, we extend the definition of $\Phi_{\mathcal{C}, \mathcal{Q}}$ into a rational map $\Phi_{\mathcal{C}_{1}, \mathcal{Q}}: \mathbb{P}^{3} \rightarrow \mathbb{P}^{3}$ given by

$$
\Phi_{\mathcal{C}_{1}, \mathcal{Q}}[x: y: z: t]=\left[\bigwedge^{3}(\nabla F(x, y, z, t) \nabla G(x, y, z, t) \nabla Q(x, y, z, t))\right],
$$

with the classical notation $\bigwedge^{3} M$ for any matrix $M \in M a t_{4 \times 3}(\mathbb{C})$ given by

$$
\bigwedge^{3} M=\left(\begin{array}{c}
-\operatorname{det} \tilde{M}^{(1)} \\
\operatorname{det} \tilde{M}^{(2)} \\
-\operatorname{det} \tilde{M}^{(3)} \\
\operatorname{det} \tilde{M}^{(4)}
\end{array}\right) \in \mathbb{C}^{4},
$$

where $\tilde{M}^{(i)}$ is the $3 \times 3$ matrix obtained from the matrix $M$ by deleting the $i$-th line.

Remark 6. The points $m \in \mathcal{C}$ for which the right hand side of (7) is not well defined are the singular points of $\mathcal{C}_{1}$ contained in $\mathcal{C}$ and the points $m \in \mathcal{C} \cap \mathcal{Q}$ such that $\mathcal{T}_{m} \mathcal{C} \subset \mathcal{T}_{m} \mathcal{Q}$.

3.2. Halphen transform of rational curves. It will be useful to consider the symmetric bilinear form $\mathfrak{b}_{Q}(\cdot, \cdot)$ on $\mathbf{W}$ associated to $Q(\cdot)$ and given on coordinates by $\mathfrak{b}_{Q}\left(\mathbf{m}_{1}, \mathbf{m}_{2}\right)=\left[Q\left(\mathbf{m}_{1}+\right.\right.$ $\left.\left.\mathbf{m}_{2}\right)-Q\left(\mathbf{m}_{1}-\mathbf{m}_{2}\right)\right] / 4$.

As $\Phi_{\mathcal{C}, \mathcal{Q}}(m)$ is in $\mathcal{T}_{m} \mathcal{C}_{1}, \Phi_{\mathcal{C}, \mathcal{Q}}(m)=\left[a \cdot \mathbf{m}+b \cdot \mathfrak{t}_{\infty, \mathcal{C}_{1}}(\mathbf{m})\right]$ for some $[a: b] \in \mathbb{P}^{1}(\mathbb{C})$. Now the fact that $\Phi_{\mathcal{C}, \mathcal{Q}}(m)$ is in $\delta_{m} \mathcal{Q}$ means that $\mathfrak{b}_{Q}\left(\mathbf{m}, \Phi_{\mathcal{C}, \mathcal{Q}}(\mathbf{m})\right)=0$, i.e. that $a \mathfrak{b}_{Q}(\mathbf{m})+b \mathfrak{b}_{Q}\left(\mathbf{m}, \mathfrak{t}_{\infty, \mathcal{C}_{1}}(\mathbf{m})\right)=$ 0 , hence we have proved the following remark.

Remark 7. If $\mathcal{C}$ is contained in a curve $\mathcal{C}_{1}=V(F, G) \subset \mathbb{P}^{3}$ and if $m \in \mathcal{C} \backslash\left(\operatorname{Sing}\left(\mathcal{C}_{1}\right) \cup \mathcal{H}^{\infty}\right)$, then

$$
\Phi_{\mathcal{C}, \mathcal{Q}}(m)=\left[\mathfrak{b}_{Q}\left(\mathbf{m}, \mathfrak{t}_{\infty, \mathcal{C}_{1}}(\mathbf{m})\right) \cdot \mathbf{m}-Q(\mathbf{m}) \cdot \mathfrak{t}_{\infty, \mathcal{C}_{1}}(\mathbf{m})\right]
$$


In particular, if $u \mapsto[\alpha(u)]$ is a local parametrization of $\mathcal{C}$ around a generic $m_{0} \in \mathcal{C}$, then a local parametrization of $\mathcal{C}^{\mathcal{Q}}$ around $\Phi_{\mathcal{C}, \mathcal{Q}}\left(m_{0}\right)$ is given by

$$
\Psi_{\mathcal{C}, \mathcal{Q}}: u \mapsto \Phi_{\mathcal{C}, \mathcal{Q}}(\alpha(u))=\left[\mathfrak{b}_{Q}\left(\alpha(u), \alpha^{\prime}(u)\right) \cdot \alpha(u)-Q(\alpha(u)) \cdot \alpha^{\prime}(u)\right] .
$$

Observe that Halphen transforms are preserved by linear isomorphisms. It is also worth noticing that the only fixed points of $\left(\Phi_{\mathcal{C}, \mathcal{Q}}\right)_{\mid \mathcal{C}}$ are in $\mathcal{C} \cap \mathcal{Q}$.

\section{Degree of the Halphen transform}

4.1. Proof of the degree formula of Theorem 1, The following result will be proved in Section 7.

Proposition 8. Let $\mathcal{C}$ be an irreducible curve of $\mathbb{P}^{3}$. Then, for a generic $\mathcal{Q}$, the map $\left(\Phi_{\mathcal{C}, \mathcal{Q}}\right)_{\mid \mathcal{C}}$ is birational.

As a consequence we obtain the following generic result.

Corollary 9. If $\mathcal{C}=V(F, G)$ is an irreducible and smooth algebraic curve of $\mathbb{P}^{3}$, then for a generic quadric $\mathcal{Q}, \operatorname{deg} \mathcal{C}^{\mathcal{Q}}=\operatorname{deg} F \times \operatorname{deg} G \times(\operatorname{deg} F+\operatorname{deg} G-1)$.

We will write $i_{m}(\mathcal{A}, \mathcal{B})$ for the intersection number of a curve $\mathcal{A} \subset \mathbb{P}^{3}$ and a surface $\mathcal{B} \subset \mathbb{P}^{3}$ at $m$. Theorem 1 is a consequence of the following result involving some polar sufaces [2].

Proposition 10. Let $\mathcal{C}$ be an algebraic curve of $\mathbb{P}^{3}$ contained in a curve $\mathcal{C}_{1}$ which is the complete intersection $V(F, G)$ of two algebraic surfaces. Assume that $\mathcal{C}$ is irreducible. Then, for a generic quadric $\mathcal{Q}$, the degree of the Halphen transform of $\mathcal{C}$ with respect to $\mathcal{Q}$ is given by

$$
\operatorname{deg} \mathcal{C}^{\mathcal{Q}}=\operatorname{deg} \mathcal{C} \times(\operatorname{deg} F+\operatorname{deg} G-1)-\sum_{m \in \mathcal{C} \cap \operatorname{Sing} \mathcal{C}_{1}} i_{m}\left(\mathcal{C}, \mathcal{P}_{\mathcal{C}_{1}, B}\right),
$$

for a generic $B \in\left(\mathbb{C}^{6}\right)^{\vee}$, where $\operatorname{Sing} \mathcal{C}_{1}$ is the set of singular points of $\mathcal{C}_{1}$ and where $\mathcal{P}_{\mathcal{C}_{1}, B}$ is the polar surface of $\mathcal{C}_{1}$ given by $\mathcal{P}_{\mathcal{C}_{1}, B}:=B\left(\bigwedge^{2}(\nabla F \nabla G)\right)$.

Proof. We use the following classical degree formula (valid for a generic quadric $\mathcal{Q}$ and a generic $\left.A \in\left(\mathbb{C}^{4}\right)^{\vee}\right):$

$$
\operatorname{deg} \mathcal{C}^{\mathcal{Q}}=\operatorname{deg} \mathcal{C} \times(\operatorname{deg} F+\operatorname{deg} G-1)-\sum_{m \in \mathcal{E}} i_{m}\left(\mathcal{C}, \mathcal{P}_{\mathcal{C}, \mathcal{Q}, A}\right),
$$

where $\mathcal{E}$ is the set of $m \in \mathcal{C}$ for which $\bigwedge^{3}(\nabla F(\mathbf{m}) \nabla G(\mathbf{m}) \nabla Q(\mathbf{m}))=\mathbf{0}$ and where $\mathcal{P}_{\mathcal{C}, \mathcal{Q}, A}$ is the Halphen polar surface given by $\mathcal{P}_{\mathcal{C}, \mathcal{Q}, A}:=V(A(\stackrel{3}{\Lambda}(\nabla F \nabla G \nabla Q))) \subset \mathbb{P}^{3}$.

Observe that, due to Remark [6, for a generic quadric $\mathcal{Q}, \mathcal{E}=\mathcal{C} \cap \operatorname{Sing} \mathcal{C}_{1}$.

Let $m \in \mathcal{C} \cap \operatorname{Sing} \mathcal{C}_{1}$. Let us prove that for generic $\mathcal{Q}, A$ and $B$, we have the equality $i_{m}\left(\mathcal{C}, \mathcal{P}_{\mathcal{C}, \mathcal{Q}, A}\right)=i_{m}\left(\mathcal{C}, \mathcal{P}_{\mathcal{C}_{1}, B}\right)$. Without loss of generality we assume that $m[0: 0: 0: 1]$. Let $\mathcal{B}$ be a branch of $\mathcal{C}$ at $m$ parametrized by some $\alpha=\left[\alpha^{(x)}: \alpha^{(y)}: \alpha^{(z)}: \alpha^{(t)}\right]$ with $\alpha^{(t)} \equiv 1$ of the form (14). For a generic quadric $\mathcal{Q}$ and a generic $A \in\left(\mathbb{P}^{3}\right)^{\vee}$, we have

$$
\begin{aligned}
& i_{m}\left(\mathcal{B}, \mathcal{P}_{\mathcal{C}, \mathcal{Q}, A}\right)= \min _{i, j, k \in\{x, y, z, t\} \text { pairwise distinct }} \min \left(\operatorname{val}\left(\alpha^{(i)} \times\left[F_{j} G_{k}-F_{k} G_{j}\right] \circ \alpha\right),\right. \\
&\left.\operatorname{val}\left(\alpha^{(i)} \times\left[F_{i} G_{k}-F_{k} G_{i}\right] \circ \alpha-\alpha^{(j)} \times\left[F_{k} G_{j}-F_{j} G_{k}\right] \circ \alpha\right)\right) .
\end{aligned}
$$


Observe first that

$$
\min _{j, k \in\{x, y, z, t\} \text { pairwise distinct }} \operatorname{val}\left(\left(F_{j} G_{k}-F_{k} G_{j}\right) \circ \alpha\right) \leq i_{m}\left(\mathcal{B}, \mathcal{P}_{\mathcal{C}, \mathcal{Q}, A}\right) .
$$

Let us prove that this inequality is indeed an equality. Since $\alpha^{(t)} \equiv 1$, we observe that

$$
i_{m}\left(\mathcal{B}, \mathcal{P}_{\mathcal{C}, \mathcal{Q}, A}\right) \leq \min _{j, k \in\{x, y, z\} \text { pairwise distinct }} \operatorname{val}\left(\left(F_{j} G_{k}-F_{k} G_{j}\right) \circ \alpha\right) .
$$

Now, if $\operatorname{val}\left(\left(F_{t} G_{\ell}-F_{\ell} G_{t}\right) \circ \alpha\right)<\min _{j, k \in\{x, y, z\} \text { pairwise distinct }} \operatorname{val}\left(\left(F_{j} G_{k}-F_{k} G_{j}\right) \circ \alpha\right)$, then

$$
\operatorname{val}\left(\alpha^{(t)} \times\left[F_{t} G_{\ell}-F_{\ell} G_{t}\right] \circ \alpha-\alpha^{(j)} \times\left[F_{\ell} G_{j}-F_{j} G_{\ell}\right] \circ \alpha\right)=\operatorname{val}\left(\left(F_{t} G_{\ell}-F_{\ell} G_{t}\right) \circ \alpha\right),
$$

for any $j \in\{x, y, z\} \backslash\{\ell\}$. Hence

$$
\begin{aligned}
i_{m}\left(\mathcal{B}, \mathcal{P}_{\mathcal{C}, \mathcal{Q}, A}\right) & =\min _{j, k \in\{x, y, z, t\}} \operatorname{pairwise} \text { distinct } \\
& =i_{m}\left(\mathcal{B}, \mathcal{P}_{\mathcal{C}_{1}, B}\right),
\end{aligned}
$$

for a generic $B \in\left(\mathbb{C}^{6}\right)^{\vee}$. This ends the proof of Theorem 10

Recall that $\operatorname{deg}_{\mathbb{P}^{5}} \mathbb{T} \mathcal{C}=\operatorname{deg} \overline{\tilde{\vartheta}_{\mathcal{C}_{1}}(\mathcal{C})}$ with $\tilde{\vartheta}_{\mathcal{C}_{1}}: \mathbb{P}^{3} \rightarrow \mathbb{P}^{5}$ given by $\tilde{\boldsymbol{\vartheta}}_{\mathcal{C}_{1}}(\mathbf{m})=\left[{ }^{2}(\nabla F(\mathbf{m}) \nabla G(\mathbf{m}))\right] \in$ $\mathbb{C}^{6}$. Since $\left(\tilde{\vartheta}_{\mathcal{C}_{1}}\right)_{\mid \mathcal{C}}$ is birational, we also have

$$
\operatorname{rank} \mathcal{C}=\operatorname{deg} \tilde{\vartheta}_{\mathcal{C}_{1}}(\mathcal{C})=\operatorname{deg} \mathcal{C}(\operatorname{deg} F+\operatorname{deg} G-2)-\sum_{m \in \mathcal{C} \cap \operatorname{Sing} \mathcal{C}_{1}} i_{m}\left(\mathcal{C}, \mathcal{P}_{\mathcal{C}_{1}, B}\right),
$$

for a generic $B \in\left(\mathbb{C}^{6}\right)^{\vee}$.

Proof of Theorem 1] We combine (9) and (11).

\subsection{Examples.}

Example 11. Consider the Viviani curve $\mathcal{V}=V\left(x^{2}+y^{2}+z^{2}-t^{2}, x^{2}-x t+y^{2}\right)$ in $\mathbb{P}^{3}$. In this case $\mathcal{C}_{1}=\mathcal{C}$ and (11) becomes $\operatorname{rank} \mathcal{V}=8-i_{P}\left(\mathcal{V}, \mathcal{P}_{\mathcal{V}, B}\right)$ with $P[1: 0: 0: 1]$. Observe that $P$ is a singular point of $\mathcal{V}$ of multiplicity 2. The tangent cone of $\mathcal{V}$ at $P$ is $V\left(y^{2}-z^{2}, x-t\right)$. Moreover the tangent plane to $\mathcal{P}_{\mathcal{V}, B}$ at $P$ is $V\left(\left(b_{1}-b_{5}\right) y+\left(b_{2}+b_{6}\right) z\right)$. Hence $\mathcal{P}_{\mathcal{V}, B}$ is always transverse to $\mathcal{V}$ at $P$ and $i_{P}\left(\mathcal{V}, \mathcal{P}_{\mathcal{V}, B}\right)=2$ (for a generic $\left.B \in\left(\mathbb{C}^{6}\right)^{\vee}\right)$. So rank $\mathcal{V}=6$ and $\operatorname{deg} \mathcal{V}^{\mathcal{Q}}=4+6=10$, for a generic quadric $\mathcal{Q}$.

Example 12. For the twisted cubic curve $\mathcal{K}=V\left(y^{2}-z x, y z-x t, y t-z^{2}\right) \subset \mathcal{C}_{1}=V\left(y^{2}-z x, y z-\right.$ $x t)$ in $\mathbb{P}^{3}$ considered in Example 14, (11) becomes $\operatorname{rank} \mathcal{C}=6-i_{P}\left(\mathcal{K}, \mathcal{P}_{\mathcal{K}, B}\right)$, with $P[0: 0: 0: 1]$. Recall that $\mathcal{C}_{1}=\mathcal{L} \cup \mathcal{K}$ where $\mathcal{L}=V(x, y)$ in $\mathbb{P}^{3}$. Observe that $P$ is a non singular point of $\mathcal{K}$ with tangent line $\mathcal{L}$ and with osculating plane $V(x)$. The tangent plane to $\mathcal{P}_{\mathcal{C}_{1}, B}$ at $P$ is $V\left(2 b_{1} y-b_{2} x\right)$ which is tangent but generically not osculating to $\mathcal{K}$ at $P$. Hence $i_{P}\left(\mathcal{K}, \mathcal{P}_{\mathcal{C}_{1}, B}\right)=2$ (for a generic $\left.B \in\left(\mathbb{C}^{6}\right)^{\vee}\right)$. We obtain $\operatorname{rank} \mathcal{K}=4$ and $\operatorname{deg} \mathcal{K}^{\mathcal{Q}}=3+4=7$, for a generic quadric $\mathcal{Q}$.

4.3. Rational curves. Consider a rational curve parametrized by a morphism $\gamma: \mathbb{P}^{1} \rightarrow \mathbb{P}^{3}$. Due to (8), $\mathcal{C}^{\mathcal{Q}}$ is the image of the rational map $\psi: \mathbb{P}^{1} \rightarrow \mathbb{P}^{3}$ given by $\psi_{\mathcal{C}, \mathcal{Q}}:=\left[\mathfrak{b}_{Q}\left(\gamma, \gamma_{u}\right) \cdot \gamma-\right.$ $\left.\mathfrak{b}_{Q}(\gamma, \gamma) \cdot \gamma_{u}\right]$. Using twice the Euler formula for $\gamma$, we obtain that

$$
\psi_{\mathcal{C}, \mathcal{Q}}=\left[\mathfrak{b}_{Q}\left(\gamma, \gamma_{u}\right) \cdot \gamma_{v}-\mathfrak{b}_{Q}\left(\gamma, \gamma_{v}\right) \cdot \gamma_{u}\right]
$$

Moreover, via the Plücker embedding, $\mathbb{T C}$ corresponds to the Zariski closure of the image of the morphism $\eta: \mathbb{P}^{1} \rightarrow \mathbb{P}^{5}$ defined on coordinates by $\eta:=\left[\bigwedge^{2}\left(\gamma \gamma_{u}\right)\right]=\left[\bigwedge^{2}\left(\gamma_{u} \gamma_{v}\right)\right]$ (since $\left.\gamma=u \gamma_{u}+v \gamma_{v}\right)$. Hence we have proved the following result. 
Proposition 13. For a generic space rational curve $\mathcal{C}$ of degree $d, \operatorname{rank} \mathcal{C}=2 d-2$ and, for a generic quadric $\mathcal{Q}$, the degree of $\mathcal{C}^{\mathcal{Q}}$ is $3 d-2$.

This is true for any smooth rational curve image of some morphism $\gamma: \mathbb{P}^{1} \rightarrow \mathbb{P}^{3}$ such that the coordinates of $\stackrel{2}{\wedge}\left(\gamma_{u} \gamma_{v}\right)$ have no common prime factor.

Example 14. For a generic quadric $\mathcal{Q}$, the Halphen transform of the twisted cubic curve $\mathcal{K}$ (image of the morphism $\gamma: \mathbb{P}^{1} \rightarrow \mathbb{P}^{3}$ given in coordinates by $\gamma(u, v)=\left(u^{3}, u^{2} v, u v^{2}, v^{3}\right)$ ) has degree \%. Moreover rank $\mathcal{K}=4$ (as already obtained in Example 12).

\section{BRANCH DESINGULARIZATION}

5.1. General case. Consider a branch $\mathcal{B}$ of type $(e, r, s)$ of $\mathcal{C}$ at $m_{0}$. Up to a linear change of variables, we assume that $m_{0}[0: 0: 0: 1]$ and that a parametrization of $\mathcal{B}$ is given by

$$
\alpha: u \mapsto\left[u^{e} \sum_{n \geq 0} a_{n} u^{n}: u^{r} \sum_{k \geq 0} b_{k} u^{k}: u^{s} \sum_{\ell \geq 0} c_{\ell} u^{\ell}: 1\right],
$$

with $0<e<r<s$ and $a_{0}=b_{0}=c_{0}=1$. Observe that the components of $\alpha$ are in $\mathbb{C}[[u]]$. We then define

$$
n_{0}:=\inf \left\{n \geq 1: a_{n} \neq 0\right\}, \quad k_{0}:=\inf \left\{k \geq 1: b_{k} \neq 0\right\} \quad \text { and } \quad \ell_{0}:=\inf \left\{\ell \geq 1: c_{\ell} \neq 0\right\} .
$$

We assume (without loss of generality) that $n_{0} \neq r$ and that $k_{0} \neq s$.

The following proposition determines the type of the branch $\Phi_{\mathcal{C}, \mathcal{Q}}(\mathcal{B})$ for every branch $\mathcal{B}$ of $\mathcal{C}$ and for a generic quadric $\mathcal{Q}$ of $\mathbb{P}^{3}$.

Proposition 15. Let $\mathcal{C}$ be a curve of $\mathbb{P}^{3}$. Let $\mathcal{B}$ be a branch of type $(e, r, s)$ of $\mathcal{C}$ with parametrization (13).

If $r \neq 2 e$ and $s \neq 2 e$, then for a generic quadric $\mathcal{Q}, \Phi_{\mathcal{C}, \mathcal{Q}}(\mathcal{B})$ is a branch of type $(a, b, c)$ with $\{a, b, c\}=\{e, r-e, s-e\}$.

If $r=2 e$ and if $s \neq \min \left(\operatorname{val}\left(\alpha_{1}^{2}-\alpha_{2}\right), 3 e\right)$, then for a generic quadric $\mathcal{Q}, \Phi_{\mathcal{C}, \mathcal{Q}}(\mathcal{B})$ is a branch of type $(a, b, c)$ with $\{a, b, c\}=\left\{e, \min \left(2 e, \operatorname{val}\left(\alpha_{1}^{2}-\alpha_{2}\right)-e\right), s-e\right\}$.

If $r=2 e$ and if $s=\operatorname{val}\left(\alpha_{1}^{2}-\alpha_{2}\right)<3 e$, then for a generic quadric $\mathcal{Q}, \Phi_{\mathcal{C}, \mathcal{Q}}(\mathcal{B})$ is a branch of type $\left(e, s-e, \min \left(2 e, \operatorname{val}\left(\alpha_{1}^{2}-\alpha_{2}-\gamma \alpha_{3}\right)-e\right)\right)$, with $\gamma:=\sum_{k=0}^{s} a_{k} a_{s-k}-b_{s}$.

If $r=2 e$ and if $s=3 e \leq \operatorname{val}\left(\alpha_{1}^{2}-\alpha_{2}\right)$, then for a generic quadric $\mathcal{Q}, \Phi_{\mathcal{C}, \mathcal{Q}}(\mathcal{B})$ is a branch of type $\left(e, 2 e, \min \left(3 e, \operatorname{val}\left(\alpha_{1}^{2}-\alpha_{2}-\gamma \alpha_{3}\right)-e, \operatorname{val}\left(\alpha_{1} \alpha_{2}-2 \alpha_{3}\right)-e, \operatorname{val}\left(\alpha_{1}^{\prime} \alpha_{2}-2 \alpha_{2}^{\prime} \alpha_{1}+\alpha_{3}^{\prime}\right)-e+1\right)\right)$.

If $s=2 e$, then for a generic quadric $\mathcal{Q}, \Phi_{\mathcal{C}, \mathcal{Q}}(\mathcal{B})$ is a branch of type $\left(r-e, e, \min \left(\operatorname{val}\left(\alpha_{1}^{2}-\right.\right.\right.$ $\left.\left.\left.\alpha_{3}\right)-e, 2 e\right)\right)$.

Corollary 16 (Singularity and inflexion decrease). We observe that for a generic quadric $\mathcal{Q}$, $\Phi_{\mathcal{C}, \mathcal{Q}}$ transforms a branch $\mathcal{B}$ of type $(e, r, s)$ in a branch of type $\left(e^{\prime}, r^{\prime}, s^{\prime}\right)$ with $e^{\prime} \leq e, r^{\prime} \leq r$ and $s^{\prime} \leq s$ and that $\left(e^{\prime}, r^{\prime}, s^{\prime}\right) \neq(e, r, s)$ except if $r=2 e$ and $s=3 e$ and

$$
4 e=\operatorname{val}\left(\alpha_{1} \alpha_{2}-2 \alpha_{3}\right)=\operatorname{val}\left(\alpha_{1}^{2}-\alpha_{2}-\gamma \alpha_{3}\right)=\operatorname{val}\left(\alpha_{1}^{\prime} \alpha_{2}-2 \alpha_{2}^{\prime} \alpha_{1}+\alpha_{3}^{\prime}\right)+1 .
$$

In particular, if $\mathcal{B}$ is an inflectional nonsingular branch of $\mathcal{C}$ of type $(1, r, s)$, then, for a generic quadric $\mathcal{Q} \subset \mathbb{P}^{3}$, the type of $\Phi_{\mathcal{C}, \mathcal{Q}}(\mathcal{B})$ is $(1, r-1, s-1)$ (due to the first case in Proposition $[15)$.

Proof of Proposition 15. Let us write $\alpha_{1}, \alpha_{2}, \alpha_{3}, \alpha_{4}$ (resp. $\psi_{1}, \psi_{2}, \psi_{3}, \psi_{4}$ ) for the four coordinates of $\alpha$ given by (13) (resp. of $\Psi$ given by (8) ). Recall that $Q(m)={ }^{t} m \cdot M \cdot m$ for some symmetric 
matrix $M=\left(m_{i, j}\right)_{i, j}$. We assume that $m_{4,4}=1$ and $m_{1,4} m_{2,4} m_{3,4} \neq 0$. With these notations we have

$$
\psi_{i_{0}}(u)=\sum_{j \neq i_{0}} A_{j}(u)\left[\alpha_{j}^{\prime}(u) \alpha_{i_{0}}(u)-\alpha_{j}(u) \alpha_{i_{0}}^{\prime}(u)\right],
$$

with $A_{j}(u):=\sum_{i} m_{i, j} \alpha_{i}(u)$. Up to a change of variable, a parametrization of $\Phi_{\mathcal{C}, \mathcal{Q}}(\mathcal{B})$ is given by $\left[\theta_{1}: \theta_{2}: \theta_{3}: \theta_{4}\right]$ with $\theta_{1}:=m_{1,4} \psi_{1}+\psi_{4}+m_{2,4} \psi_{2}+m_{3,4} \psi_{3}, \theta_{2}:=\psi_{2}, \theta_{3}:=\psi_{3}$ and $\theta_{4}:=\psi_{4}$. We have

$\theta_{1}=B_{1,4} \alpha_{1}^{\prime}+B_{2,4} \alpha_{2}^{\prime}+B_{3,4} \alpha_{3}^{\prime}+B_{2,1}\left(\alpha_{2}^{\prime} \alpha_{1}-\alpha_{2} \alpha_{1}^{\prime}\right)+B_{3,1}\left(\alpha_{3}^{\prime} \alpha_{1}-\alpha_{3} \alpha_{1}^{\prime}\right)+B_{3,2}\left(\alpha_{3}^{\prime} \alpha_{2}-\alpha_{3} \alpha_{2}^{\prime}\right)$, with $B_{i, j}:=m_{j, 4} A_{i}-m_{i, 4} A_{j}$. Observe that val $B_{i, j}=$ val $\alpha_{1}=e$ and so val $\theta_{1}=2 e-1$. Moreover $\operatorname{val} \theta_{2}=r-1, \operatorname{val} \theta_{3}=s-1$ and $\operatorname{val} \theta_{4}=e-1$. Observe that Then $\operatorname{val}\left(\theta_{1}\right)=\operatorname{val}\left(\theta_{2}\right)=2 e-1$ and

$$
\begin{gathered}
\theta_{1}(u)=\left(m_{1,1}-m_{1,4}^{2}\right) \alpha_{1} \alpha_{1}^{\prime}+(e+r)\left(m_{1,2}-m_{1,4} m_{2,4}\right) u^{r+e-1}+h_{1}(u), \\
\theta_{2}(u)=-\alpha_{2}^{\prime}(u)+m_{1,4}(e-2 r) u^{e+r-1}+h_{2}(u), \\
\theta_{3}(u)=-\alpha_{3}^{\prime}(u)+m_{1,4}(e-2 s) u^{e+s-1}+h_{3}(u),
\end{gathered}
$$

with val $h_{1}$, val $h_{2}>r+e-1$ and val $h_{3}>s+e-1$.

- If $e, r-e$ and $s-e$ are pairwise distinct, $\Phi_{\mathcal{C}, \mathcal{Q}}(\mathcal{B})$ is a branch of type $(a, b, c)$ with $\{a, b, c\}=\{e, r-e, s-e\}$ and $a<b<c$.

- Assume now that $r=2 e$, i.e. $e=r-e<s-e$ and

$$
\begin{aligned}
\tilde{\theta}_{1}(u) & :=\frac{2}{m_{1,1}-m_{1,4}^{2}} \theta_{1}(u)+\theta_{2}(u) \\
& =\left(\alpha_{1}^{2}-\alpha_{2}\right)^{\prime}(u)+6 e \frac{m_{1,2}-m_{1,4} m_{2,4}}{m_{1,1}-m_{1,4}^{2}} u^{3 e-1}+h(u)
\end{aligned}
$$

with val $h>3 e-1$ and

$$
v_{1}:=\operatorname{val}\left(2 \theta_{1}+\left(m_{1,1}-m_{1,4}^{2}\right) \theta_{2}\right)=\min \left(\operatorname{val}\left(\alpha_{1}^{2}-\alpha_{2}\right)^{\prime}, 3 e-1\right) .
$$

- If $v_{1} \neq s-1$, we conclude that $\Phi_{\mathcal{C}, \mathcal{Q}}(\mathcal{B})$ is a branch of type $(a, b, c)$ with $\{a, b, c\}=$ $\left\{e, v_{1}-e+1, s-e\right\}$ and $a<b<c$.

- If $v_{1}=s-1<3 e-1$, then

$$
v_{2}:=\operatorname{val}\left(\tilde{\theta}_{1}+\gamma \theta_{3}\right)=\min \left(\operatorname{val}\left(\alpha_{1}^{2}-\alpha_{2}-\gamma \alpha_{3}\right)-1,3 e-1\right)>s-1
$$

(since $e+s-1>3 e-1)$. We conclude that $\Phi_{\mathcal{C}, \mathcal{Q}}(\mathcal{B})$ is a branch of type $(e, s-$ $\left.e, \min \left(2 e, v_{2}-e+1\right)\right)$.

- If $v_{1}=s-1=3 e-1$, then val $\tilde{\theta}_{1}=\operatorname{val} \theta_{3}=3 e-1>\operatorname{val} \theta_{2}=2 e-1$ and

$$
\begin{aligned}
& \tilde{\theta}_{2}:=\tilde{\theta}_{1}+\left[2 \frac{m_{1,2}-m_{1,4} m_{2,4}}{m_{1,1}-m_{1,4}^{2}}+\gamma-m_{1,4}\right] \theta_{3} \\
&=\left(\alpha_{1}^{2}-\alpha_{2}-\gamma \alpha_{3}\right)^{\prime}+2 \frac{m_{1,2}-m_{1,4} m_{2,4}}{m_{1,1}-m_{1,4}^{2}}\left(\alpha_{1}^{\prime} \alpha_{2}+\alpha_{1} \alpha_{2}^{\prime}-\alpha_{3}^{\prime}\right)+m_{1,4}\left(\alpha_{1}^{\prime} \alpha_{2}-2 \alpha_{1} \alpha_{2}^{\prime}+\alpha_{3}^{\prime}\right) \\
&+\left[2 \frac{m_{1,4} m_{1,2}-m_{1,1} m_{2,4}}{m_{1,1}-m_{1,4}^{2}}-m_{1,1}\right] \alpha_{1}\left(\alpha_{2}^{\prime} \alpha_{1}-\alpha_{2} \alpha_{1}^{\prime}\right)+\left[2 \frac{m_{2,2}-m_{2,4}^{2}}{m_{1,1}-m_{1,4}^{2}}-m_{2,4}\right] \alpha_{2} \alpha_{2}^{\prime} \\
& \quad+2 \frac{m_{1,3}-m_{1,4} m_{3,4}}{m_{1,1}-m_{1,4}^{2}}\left(\alpha_{3} \alpha_{1}^{\prime}-\alpha_{1} \alpha_{3}^{\prime}\right)+\tilde{h}(u),
\end{aligned}
$$

with val $\tilde{h} \geq 4 e$. Hence

$\operatorname{val} \tilde{\theta}_{2}=\min \left(\operatorname{val}\left(\alpha_{1}^{2}-\alpha_{2}-\gamma \alpha_{3}\right)-1, \operatorname{val}\left(\alpha_{1} \alpha_{2}-2 \alpha_{3}\right)-1, \operatorname{val}\left(\alpha_{1}^{\prime} \alpha_{2}-2 \alpha_{2}^{\prime} \alpha_{1}+\alpha_{3}^{\prime}\right), 4 e-1\right)$.

We conclude that $\Phi_{\mathcal{C}, \mathcal{Q}}(\mathcal{B})$ is a branch of type $\left(e, 2 e, \operatorname{val} \tilde{\theta}_{2}-e+1\right)$. 
- Assume that $r-e<s-e=e$. Then $\operatorname{val} \theta_{2}=r-1<\operatorname{val} \theta_{1}=\operatorname{val} \theta_{3}=2 e-1$. We already now that the type of $\Phi_{\mathcal{C}, \mathcal{Q}}(\mathcal{B})$ starts with $r-e$. As above, we observe that

$$
\begin{aligned}
v_{3} & :=\operatorname{val}\left(2 \theta_{1}+\left(m_{1,1}-m_{1,4}^{2}\right) \theta_{3}\right) \\
& =\min \left(\operatorname{val}\left(\alpha_{1}^{2}-\alpha_{3}\right)^{\prime}, 3 e-1\right)>2 e-1
\end{aligned}
$$

and we conclude that $\Phi_{\mathcal{C}, \mathcal{Q}}(\mathcal{B})$ is a branch of type $\left(r-e, e, v_{3}-e+1\right)$.

This ends the proof of Proposition 15 .

5.2. Desingularization via iteration of Halphen maps. Recall that a branch is smooth if it has type $(1,2,3)$. Observe that, by generic Halphen transforms, a curve of type $(e, r=e+1, s)$ with $1 \neq e<r<s$ becomes a curve of type $\left(1, e, s_{1}\right)$ with $s_{1} \leq \min (s, 2 e)\left(s_{1}=s-e\right.$ if $\left.s \neq 2 e\right)$. Moreover, a curve of type $\left(1, e, s_{1}\right)$ becomes a curve of type $\left(1,2, s_{1}-e+2\right)$ in $(e-2)$ steps and a curve of type $\left(1,2, s_{1}-e+2\right)$ becomes a curve of type $(1,2,3)$ in $\left(s_{1}-e-1\right)$ steps. Hence it is desingularized in at most $s_{1}+1$ steps.

Some desingularizations by generic Halphen transforms are summarized in the following scheme on which each arrow corresponds to the Halphen transform for a generic quadric $\mathcal{Q}$ of $\mathbb{P}^{3}$ :

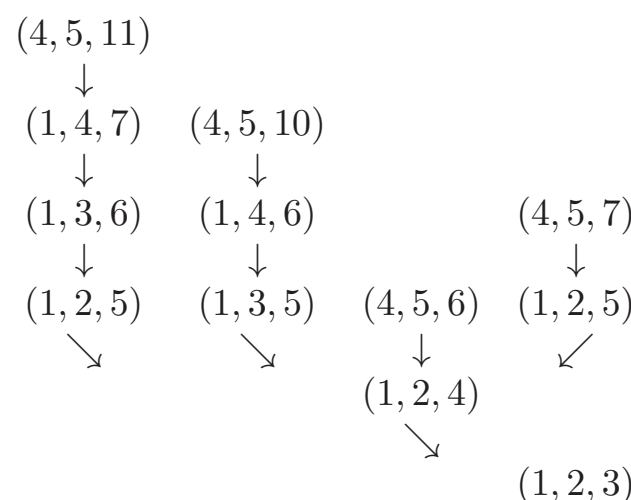

\section{RANK AND Class of the Halphen transform}

6.1. Proof of the formulas of Theorem 1. Let $\mathcal{C}$ be a non-planar irreducible curve of $\mathbb{P}^{3}$. To understand Piene's formula for the rank of $\mathcal{C}$, let us recall the notion of type of a branch $\mathcal{B}$ at $m_{0} \in \mathcal{C}$. We say that the branch $\mathcal{B}$ has type $(e, r, s)$ if, up to a linear change of coordinates, $m_{0}[0: 0: 0: 1]$ and $\mathcal{B}$ is parametrized by

$$
\alpha: u \mapsto\left[u^{e} \sum_{n \geq 0} a_{n} u^{n}: u^{r} \sum_{k \geq 0} b_{k} u^{k}: u^{s} \sum_{\ell \geq 0} c_{\ell} u^{\ell}: 1\right],
$$

with $0<e<r<s$ and $a_{0}=b_{0}=c_{0}=1$. Recall that $e$ is the multiplicity of $\mathcal{B}$ and that $s$ is the intersection multiplicity of $\mathcal{B}$ with its osculating plane. Then the two first stationnary indices of $\mathcal{B}$ are given by the following formulas

$$
k_{0}(\mathcal{B})=e-1 \quad \text { and } \quad k_{1}(\mathcal{B})=r-e-1
$$

Observe that, if $\mathcal{B}$ is smooth and ordinary (since in this case $\mathcal{B}$ has type $(1,2,3)$ ), then $k_{0}(\mathcal{B})=$ $k_{1}(\mathcal{B})=0$.

The stationnary indices $k_{i}(\mathcal{C})$ is the sum of the $k_{i}(\mathcal{B})$ over the set of branches $\mathcal{B}$ of $\mathcal{C}$. The 0 -th stationnary index $k_{0}(\mathcal{C})$ corresponds to the number of cusps of $\mathcal{C}$ (computed with their multiplicities). The first stationnary index $k_{1}(\mathcal{C})$ corresponds to the number of (possibly singular) 
inflection points of $\mathcal{C}$ (computed with their multiplicities). In [9, Example (3.2)] (see also [10, Section 2]), Piene established the following formulas for the rank and the class of $\mathcal{C}$ :

$$
\begin{gathered}
\operatorname{rank} \mathcal{C}=2[\operatorname{deg} \mathcal{C}+g(\mathcal{C})-1]-k_{0}(\mathcal{C}), \\
\operatorname{class} \mathcal{C}=3[\operatorname{deg} \mathcal{C}+2 g(\mathcal{C})-2]-2 k_{0}(\mathcal{C})-k_{1}(\mathcal{C}) .
\end{gathered}
$$

Let $\mathcal{Q}$ be a generic quadric of $\mathbb{P}^{3}$ such that $\Phi_{\mathcal{C}, \mathcal{Q}}$ is birational and such that $\operatorname{deg} \mathcal{C}^{\mathcal{Q}}=\operatorname{deg} \mathcal{C}+$ rank $\mathcal{C}$. Then $g\left(\mathcal{C}^{\mathcal{Q}}\right)=g(\mathcal{C})$ and so, using Piene's formulas (15) and (16), we obtain

$$
\begin{aligned}
\operatorname{rank} \mathcal{C}^{\mathcal{Q}} & =2\left[\operatorname{deg} \mathcal{C}^{\mathcal{Q}}+g\left(\mathcal{C}^{\mathcal{Q}}\right)-1\right]-k_{0}\left(\mathcal{C}^{\mathcal{Q}}\right) \\
& =2[\operatorname{deg} \mathcal{C}+\operatorname{rank} \mathcal{C}+g(\mathcal{C})-1]-k_{0}\left(\mathcal{C}^{\mathcal{Q}}\right)
\end{aligned}
$$

and

$$
\begin{aligned}
\operatorname{class} \mathcal{C}^{\mathcal{Q}} & =3\left[\operatorname{deg} \mathcal{C}^{\mathcal{Q}}+2 g\left(\mathcal{C}^{\mathcal{Q}}\right)-2\right]-2 k_{0}\left(\mathcal{C}^{\mathcal{Q}}\right)-k_{1}\left(\mathcal{C}^{\mathcal{Q}}\right) \\
& =3[\operatorname{deg} \mathcal{C}+\operatorname{rank} \mathcal{C}+2 g(\mathcal{C})-2]-2 k_{0}\left(\mathcal{C}^{\mathcal{Q}}\right)-k_{1}\left(\mathcal{C}^{\mathcal{Q}}\right)
\end{aligned}
$$

Now we can use Proposition 15 to compute $k_{0}\left(\mathcal{C}^{\mathcal{Q}}\right)$ and $k_{1}\left(\mathcal{C}^{\mathcal{Q}}\right)$.

\subsection{Application to examples.}

Example 17 (Halphen transform of a rational sextic). Consider the sextic curve $\mathcal{C}=V\left(z t^{2}-\right.$ $\left.x^{3}-x y t-y^{2} t, z t-x y+y^{2}\right) \subset \mathbb{P}^{3}$ intersection of a non singular cubic $\mathcal{K}$ with a tangential sphere $\mathcal{S}$. Then

and

$$
\operatorname{deg} \mathcal{C}=6, \quad g(\mathcal{C})=0, \operatorname{rank}(\mathcal{C})=7, \operatorname{class} \mathcal{C}=6
$$

$$
\operatorname{deg} \mathcal{C}^{\mathcal{Q}}=13, \quad g\left(\mathcal{C}^{\mathcal{Q}}\right)=0, \operatorname{rank}\left(\mathcal{C}^{\mathcal{Q}}\right)=24, \operatorname{class} \mathcal{C}^{\mathcal{Q}}=32
$$

Proof. This curve is the image of the map $\gamma: \mathbb{P}^{1} \rightarrow \mathbb{P}^{3}$ given by

$$
\gamma([s: t])=\left[-t^{2} s^{4}:-\frac{\sqrt{2}}{2} t^{3} s^{3}: \frac{\sqrt{2}}{2} t^{5} s-\frac{1}{2} t^{6}: s^{6}\right] .
$$

Hence

$$
\operatorname{deg} \mathcal{C}=6 \quad \text { and } \quad g(\mathcal{C})=0
$$

The curve $\mathcal{C}$ has exactly two singular points $P_{1}[0: 0: 0: 1]$ and $P_{2}[0: 0: 1: 0]$ and no nonsingular inflection points. The curve $\mathcal{C}$ admits a single branch $\mathcal{B}_{1}$ at $P_{1}$ parametrized by $\tilde{\alpha}^{(1)}(t)=\gamma([1: t])$. This branch has type $(2,3,5)$. Hence $k_{0}\left(\mathcal{B}_{1}\right)=1$ and $k_{1}\left(\mathcal{B}_{1}\right)=0$. Moreover, due to Proposition [15, for a generic quadric $\mathcal{Q} \subset \mathbb{P}^{3}$, the type of $\Phi_{\mathcal{C}, \mathcal{Q}}\left(\mathcal{B}_{1}\right)$ is $(1,2,3)$ and so $k_{0}\left(\Phi_{\mathcal{C}, \mathcal{Q}}\left(\mathcal{B}_{1}\right)\right)=k_{1}\left(\Phi_{\mathcal{C}, \mathcal{Q}}\left(\mathcal{B}_{1}\right)\right)=0$.

Analogously $\mathcal{C}$ admits a single branch $\mathcal{B}_{2}$ at $P_{2}$ parametrized by $\tilde{\alpha}^{(2)}(s)=\gamma([s: 1])$ which can be rewritten

and so

$$
\tilde{\alpha}^{(2)}(s)=\left[\frac{2}{1-\sqrt{2} s} s^{4}: \frac{\sqrt{2}}{1-\sqrt{2} s} s^{3}: 1: \frac{-2 s^{6}}{1-\sqrt{2} s}\right]
$$

$$
\tilde{\alpha}^{(2)}(s)=\left[2 s^{4} \sum_{n \geq 0}(\sqrt{2} s)^{n}: \sqrt{2} s^{3} \sum_{n \geq 0}(\sqrt{2} s)^{n}: 1:-2 s^{6} \sum_{n \geq 0}(\sqrt{2} s)^{n}\right] .
$$

Up to a linear change of variable $\tilde{\alpha}^{(2)}$ can be replaced by the following $\alpha^{(2)}$ fitting the assumptions of our Proposition [15:

$$
\alpha^{(2)}(s)=\left[s^{3} \sum_{n \geq 0}(\sqrt{2} s)^{n}: s^{4} \sum_{n \geq 0}(\sqrt{2} s)^{n}: s^{6} \sum_{n \geq 0}(\sqrt{2} s)^{n}: 1\right] .
$$


In particular $\mathcal{B}_{2}$ has type $(3,4,6)$. So $k_{0}\left(\mathcal{B}_{2}\right)=2$ and $k_{1}\left(\mathcal{B}_{2}\right)=0$. We observe that we are in the case of a branch of type $(e, r, s)=(3,4,6)$ with $s=3 e$ and that $\left(\alpha_{1}^{(1)}(s)\right)^{2}-\alpha_{3}^{(1)}(s)=\sqrt{2} s^{7}+\ldots$ so val $\left(\left(\alpha_{1}^{(1)}\right)^{2}-\alpha_{3}^{(1)}\right)=7<9=3 e$. Therefore, due to Proposition 15, $\Phi_{\mathcal{C}, \mathcal{Q}}\left(\mathcal{B}_{2}\right)$ has type $(1,3,7)$ for a generic quadric $\mathcal{Q} \subset \mathbb{P}^{3}$. In particular $k_{0}\left(\Phi_{\mathcal{C}, \mathcal{Q}}\left(\mathcal{B}_{2}\right)\right)=0$ and $k_{1}\left(\Phi_{\mathcal{C}, \mathcal{Q}}\left(\mathcal{B}_{2}\right)\right)=1$. Hence $k_{0}(\mathcal{C})=k_{0}\left(\mathcal{B}_{1}\right)+k_{0}\left(\mathcal{B}_{2}\right)=3, k_{1}(\mathcal{C})=k_{1}\left(\mathcal{B}_{1}\right)+k_{1}\left(\mathcal{B}_{2}\right)=0, k_{0}\left(\mathcal{C}^{\mathcal{Q}}\right)=k_{0}\left(\Phi_{\mathcal{C}, \mathcal{Q}}\left(\mathcal{B}_{1}\right)\right)+$ $k_{0}\left(\Phi_{\mathcal{C}, \mathcal{Q}}\left(\mathcal{B}_{2}\right)\right)=0$ and $k_{1}\left(\mathcal{C}^{\mathcal{Q}}\right)=k_{1}\left(\Phi_{\mathcal{C}, \mathcal{Q}}\left(\mathcal{B}_{1}\right)\right)+k_{1}\left(\Phi_{\mathcal{C}, \mathcal{Q}}\left(\mathcal{B}_{2}\right)\right)=1$. Using Piene's formulas (15) and (16), we obtain that

$$
\operatorname{rank} \mathcal{C}=2[\operatorname{deg} \mathcal{C}+g(\mathcal{C})-1]-k_{0}(\mathcal{C})=2[6+0-1]-3=7
$$

and

$$
\operatorname{class} \mathcal{C}=3[\operatorname{deg} \mathcal{C}+2 g(\mathcal{C})-2]-2 k_{0}(\mathcal{C})-k_{1}(\mathcal{C})=3 \cdot 4-2 \cdot 3-0=6 .
$$

Due to Theorem 1, for a generic quadric $\mathcal{Q} \subset \mathbb{P}^{3}$, we have

$$
\begin{gathered}
\operatorname{deg} \mathcal{C}^{\mathcal{Q}}=\operatorname{deg} \mathcal{C}+\operatorname{rank} \mathcal{C}=6+7=13, \quad g\left(\mathcal{C}^{\mathcal{Q}}\right)=g(\mathcal{C})=0, \\
\operatorname{rank} \mathcal{C}^{\mathcal{Q}}=2(\operatorname{deg} \mathcal{C}+\operatorname{rank} \mathcal{C}+g(\mathcal{C})-1)-k_{0}\left(\mathcal{C}^{\mathcal{Q}}\right)=2 \cdot 12-0=24
\end{gathered}
$$

and

$$
\operatorname{class} \mathcal{C}^{\mathcal{Q}}=3 \operatorname{deg} \mathcal{C}+3 \operatorname{rank} \mathcal{C}+6 g(\mathcal{C})-6-2 k_{0}\left(\mathcal{C}^{\mathcal{Q}}\right)-k_{1}\left(\mathcal{C}^{\mathcal{Q}}\right)=3 \cdot 6+3 \cdot 7-6-1=32 .
$$

Example 18 (Halphen transform of a non rational sextic). Consider the sextic curve $\mathcal{C}=$ $V\left(x^{2} z+t z^{2}+y^{3}, x^{2}+y^{2}+z^{2}-2 z t\right) \subset \mathbb{P}^{3}$ intersection of a cubic $\mathcal{K}$ having an E6 singularity with a tangential sphere $\mathcal{S}$. Then

$$
\operatorname{deg} \mathcal{C}=6, \quad g(\mathcal{C})=1, \operatorname{rank} \mathcal{C}=10
$$

and

$$
\operatorname{deg} \mathcal{C}^{\mathcal{Q}}=16, \quad g\left(\mathcal{C}^{\mathcal{Q}}\right)=1, \operatorname{rank} \mathcal{C}^{\mathcal{Q}}=32
$$

Proof. The rational E6 cubic $\mathcal{K}$ is the image of $f: \mathbb{P}^{2} \mapsto \mathbb{P}^{3}$ given by

$$
f([u: v: w])=\left[-v^{2} w:-u v^{2}:-v^{3}: v w^{2}+u^{3}\right] .
$$

Observe that $\mathcal{C}$ is the image of $\mathcal{C}^{\prime}$ by $f$ where $\mathcal{C}^{\prime}:=V\left(3 v w^{2}+u^{2} v+v^{3}+2 u^{3}\right) \subset \mathbb{P}^{2}$. Observe also that $\mathcal{C}=V\left(x^{2}+y^{2}+z^{2}-2 z t, 3 x^{2} z+y^{2} z+z^{3}+2 y^{3}\right) \subset \mathbb{P}^{3}$ (replacing $x^{2} z+t z^{2}+y^{3}$ by $\left.2\left[x^{2} z+t z^{2}+y^{3}\right]+z\left[x^{2}+y^{2}+z^{2}-2 z t\right]\right)$.

Observe that the point $O[0: 0: 0: 1]$ is the only singular point of $\mathcal{C}$. At this point, $\mathcal{C}$ has a single branch $\mathcal{B}_{1}$ of multiplicity 3 with tangent line $V(y, z)$ and of type $(3,4,6)$ parametrized by $\tilde{\alpha}^{(1)}$ of the following form

$$
\tilde{\alpha}^{(1)}(t):=\left[\frac{\sqrt{2}}{3^{\frac{1}{4}}}\left(t^{3}-\frac{1}{2 \sqrt{3}} t^{5}-\frac{1}{48} t^{7}+O\left(t^{9}\right)\right): t^{4}: \frac{1}{\sqrt{3}} t^{6}+\frac{1}{6} t^{8}+O\left(t^{9}\right): 1\right] .
$$

Recall that $\mathcal{P}_{\mathcal{C}, B}$ has equation

$b_{1}\left(2 x y z-3 x y^{2}\right)+b_{2}\left(2 x z^{2}-4 x z t-x^{3}\right)-3 b_{3} x z^{2}+b_{4}\left(3 y^{2} z-3 y^{2} t-y x^{2}-2 y z t\right)-b_{5}\left(3 y^{2} z+y z^{2}\right)-b_{6}\left(z^{3}+z^{2} t+x^{2} z\right)$.

Using the local parametrization $\tilde{\alpha}^{(1)}$, we obtain $i_{O}\left(\mathcal{C}, \mathcal{P}_{\mathcal{C}, B}\right)=8$ (for a generic $\left.B \in\left(\mathbb{C}^{6}\right)^{\vee}\right)$ and so, due to (11), we obtain

$$
\operatorname{rank} \mathcal{C}=6(3+2-2)-8=10 \text {. }
$$

Observe that $k_{0}(\mathcal{C})=3-1=2$. Combining this with the Piene's formula (15) for the rank, we obtain that

$$
g(\mathcal{C})=\frac{1}{2}\left[\operatorname{rank}(\mathcal{C})+k_{0}(\mathcal{C})\right]+1-\operatorname{deg} \mathcal{C}=\frac{1}{2}[10+2]+1-6=1 .
$$


Hence, due to due to (11) and (2), we already know that

$$
g\left(\mathcal{C}^{\mathcal{Q}}\right)=1 \quad \text { and } \quad \operatorname{deg} \mathcal{C}^{\mathcal{Q}}=6+10=16
$$

for a generic quadric $\mathcal{Q} \subset \mathbb{P}^{3}$. Up to a linear change of coordinates, we identify $\tilde{\alpha}^{(1)}$ with $\alpha^{(1)}$ satisfying the assumptions of our Proposition 15 .

$$
\alpha^{(1)}(t):=\left[t^{3}-\frac{1}{2 \sqrt{3}} t^{5}-\frac{1}{48} t^{7}+O\left(t^{9}\right): t^{4}: t^{6}+\frac{1}{2 \sqrt{3}} t^{8}+O\left(t^{9}\right): 1\right]
$$

Observe that we are in the case of a type $(e, r, s)=(3,4,6)$ with $s=2 e$ and $\operatorname{val}\left(\left(\alpha_{1}^{(1)}\right)^{2}-\alpha_{3}^{(1)}\right)=$ $8<3 e$. So the type of the image branch is $(r-e, e, 8-e)=(1,3,5)$. Hence $k_{0}\left(\mathcal{C}^{\mathcal{Q}}\right)=0$ and so, due to (3), we obtain

$$
\operatorname{rank} \mathcal{C}^{\mathcal{Q}}=2(6+10+1-1)-0=32 .
$$

Observe moreover that if $\mathcal{B}$ is an inflectional nonsingular branch of $\mathcal{C}$, then its type is $(1, r, s)$ with $2<r<s$ and then the type of $\Phi_{\mathcal{C}, \mathcal{Q}}(\mathcal{B})$ is $(1, r-1, s-1)$ (first case in Proposition 15) which means that $k_{1}\left(\Phi_{\mathcal{C}, \mathcal{Q}}(\mathcal{B})\right)=k_{1}(\mathcal{B})-1$ whereas $k_{1}\left(\Phi_{\mathcal{C}, \mathcal{Q}}(\mathcal{B})\right)=1+k_{1}(\mathcal{B})=1$.

\section{Proof of the BIRATionality}

Observe that Proposition 8 is true if $\mathcal{C}$ is a line of $\mathbb{P}^{3}$. We will assume from now that $\mathcal{C}$ is not a line. The proof leads on the following lemmas.

Lemma 19. Let $\mathcal{C}$ be an irreducible curve of $\mathbb{P}^{3}$. Let $m_{0}$ be a nonsingular point of $\mathcal{C}$. Then, for a generic $\mathcal{Q}$, there exist no $m^{\prime} \in \mathcal{C} \backslash\left\{m_{0}\right\}$ such that $\Phi_{\mathcal{C}, \mathcal{Q}}\left(m^{\prime}\right)=\Phi_{\mathcal{C}, \mathcal{Q}}\left(m_{0}\right)$.

Proof. Since Halphen transforms are preserved by linear isomorphisms, we assume without any loss of generality that $m_{0}[0: 0: 0: 1]$ and that $t_{m_{0}} \mathcal{C}[1: 0: 0: 0]$. Recall that $\mathcal{Q}=V(Q)$ with $Q$ of the form $Q(m)={ }^{t} m \cdot M \cdot m$ for some symmetric matrix $M=\left(m_{i, j}\right)_{i, j}(M \cdot m$ corresponds to $\nabla Q / 2)$. Observe that $\Phi_{\mathcal{C}, \mathcal{Q}}\left(m_{0}\right)=\left[-m_{4,4}: 0: 0: m_{1,4}\right]$. Let $m_{1}\left[x_{1}: y_{1}: z_{1}: t_{1}\right]$ be a nonsingular point of $\mathcal{C}, \Phi_{\mathcal{C}, \mathcal{Q}}\left(m_{1}\right)=\Phi_{\mathcal{C}, \mathcal{Q}}\left(m_{0}\right)$ is equivalent to $\Phi_{\mathcal{C}, \mathcal{Q}}\left(m_{0}\right) \in \mathcal{T}_{m_{1}} \mathcal{C}$ and $\Phi_{\mathcal{C}, \mathcal{Q}}\left(m_{0}\right) \in \delta_{m_{1}} \mathcal{Q}$, i.e. to

$$
\left\{\begin{array}{c}
m_{1,4} F_{t}\left(m_{1}\right)-m_{4,4} F_{x}\left(m_{1}\right)=0, \quad m_{1,4} G_{t}\left(m_{1}\right)-m_{4,4} G_{x}\left(m_{1}\right)=0 \\
x_{1}\left(m_{1,4}^{2}-m_{1,1} m_{4,4}\right)+y_{1}\left(m_{1,4} m_{2,4}-m_{1,2} m_{4,4}\right)+z_{1}\left(m_{1,4} m_{3,4}-m_{1,3} m_{4,4}\right)=0
\end{array}\right.
$$

if $\mathcal{C}$ is contained in a curve $\mathcal{C}_{1}=V(F, G)$ of $\mathbb{P}^{3}$. Given a quadric $\mathcal{Q}$, we write $\mathcal{H}_{\mathcal{Q}}$ for the set of $\left[x_{1}: y_{1}: z_{1}: t_{1}\right] \in \mathbb{P}^{3}$ satisfying the last line of (17). Observe that, for any $a \in \mathbb{C}^{*}$ and any plane $\mathcal{H}$ containing $m_{0}$, there exists $M$ such that $m_{1,4}=a, m_{4,4}=1$ and such that $\mathcal{H}_{\mathcal{Q}}=\mathcal{H}$.

We have to prove that, for a generic $M$, no point of $\mathcal{C} \backslash\left\{m_{0}\right\}$ is solution of (17). The contrary would mean that for an infinite number of $a \in \mathbb{C}^{*}$, there exists an infinity of planes $\mathcal{H}$ passing through $m_{0}$ such that $\mathcal{C} \backslash\left\{m_{0}\right\}$ intersects $\mathcal{H} \cap V\left(a F_{t}-F_{x}, a G_{t}-G_{x}\right)$. Since $\mathcal{C}$ is irreducible, this would imply that $\mathcal{C} \subset V\left(a F_{t}-F_{x}, a G_{t}-G_{x}\right)$ for an infinity of $a$ and so that $\mathcal{C} \subset V\left(F_{t}, F_{x}, G_{t}, G_{x}\right)$. This would mean that for every $m \in \mathcal{C}, \nabla F(m)$ and $\nabla G(m)$ are contained in $V(x, t)$. But for a generic $m \in \mathcal{C}, \nabla F(m)$ and $\nabla G(m)$ are not proportional, so $t_{m} \mathcal{C} \in V(y, z)$ and so $t_{m} \mathcal{C}[1: 0: 0: 0]$. Hence $\mathcal{C}$ would be the line $V(x, t)$.

We reinforce this lemma in the following one.

Lemma 20. Let $\mathcal{C}$ be an irreducible curve of $\mathbb{P}^{3}$. There exists $N$ such that for any nonsingular point $m_{0}$ of $\mathcal{C}$, the set $\mathcal{B}_{m_{0}}$ of the quadrics $\mathcal{Q}$ such that $\# \mathcal{C} \cap\left(\Phi_{\mathcal{C}, \mathcal{Q}}^{-1}\left(\left\{m_{0}\right\}\right)\right)>1$ is contained in an hypersurface $\mathcal{K}_{m_{0}}$ of the $\mathbb{P}^{9}$ of quadrics of $\mathbb{P}^{3}$, this hypersurface has degree less than $N$. 
Proof. We use the same notations as in the preceding lemma and we suppose that $F$ and $G$ are irreducible. Recall that $\mathcal{Q}=V(Q)$ with $Q$ of the form $Q(m)={ }^{t} m \cdot M \cdot m$ for some symmetric matrix $M=\left(m_{i, j}\right)_{i, j}$. Hence we identify the set of quadrics of $\mathbb{P}^{3}$ with $\mathbb{P}^{9}$. Let us consider the following parametrization of $\mathcal{H}_{\mathcal{Q}}$ :

$$
\begin{aligned}
\psi\left(y_{1}, z_{1}, t_{1}\right):=\left(y_{1}\left(m_{1,2} m_{4,4}-m_{1,4} m_{2,4}\right)+z_{1}\left(m_{1,3} m_{4,4}-m_{1,4} m_{3,4}\right),\right. \\
\\
\left.y_{1}\left(m_{1,4}^{2}-m_{1,1} m_{4,4}\right), z_{1}\left(m_{1,4}^{2}-m_{1,1} m_{4,4}\right), t_{1}\left(m_{1,4}^{2}-m_{1,1} m_{4,4}\right)\right) .
\end{aligned}
$$

Let us write $K_{1}:=m_{1,4} F_{t} \circ \psi-m_{4,4} F_{x} \circ \psi, K_{2}:=m_{1,4} G_{t} \circ \psi-m_{4,4} G_{x} \circ \psi, K_{3}:=F \circ \psi$ and $K_{4}:=G \circ \psi$. Due to (17), $\mathcal{B}_{m_{0}}$ is contained in the algebraic variety $\mathcal{K}_{m_{0}}$ of quadrics $\mathcal{Q}$ given by the vanishing of the resultant with respect to $y_{1}$ :

$$
\forall i \in\{1,2\}, \quad \operatorname{Res}_{z_{1}}\left(z_{1}^{-1} \operatorname{Res}_{y_{1}}\left(K_{3}, K_{4}\right), \operatorname{Res}_{y_{1}}\left(K_{i}, K_{i+2}\right)\right)=0 .
$$

Let us show that a generic $\mathcal{Q}$ is not in $\mathcal{K}_{m_{0}}$ (up to a linear change of variables in $(y, z)$ ).

Since $\mathcal{C} \neq V(x, y)$, either $\mathcal{C} \not \subset V\left(F_{x}, F_{t}\right)$ or $\mathcal{C} \not \subset V\left(G_{x}, G_{t}\right)$. Assume for example that $\mathcal{C} \not \subset$ $V\left(F_{x}, F_{t}\right)$, and so $V(F)$ is not a plane. If $V(G)$ is a plane then it is of the form $V(\alpha . x+\beta . t)$. Hence, for a generic $\mathcal{Q}, V\left(K_{1}\right)$ does not contain a line and if $V\left(K_{2}\right)$ contains a line then this line has not the form $V\left(a . z_{1}+b . t_{1}\right)$.

For a generic $\mathcal{Q}, \mathcal{C} \cap \mathcal{H}_{\mathcal{Q}} \subset \mathbb{P}^{3}$ is finite, so are $V\left(K_{3}, K_{4}\right) \subset \mathbb{P}^{2}$ and $V\left(\operatorname{Res}_{y_{1}}\left(K_{3}, K_{4}\right)\right) \subset \mathbb{P}^{1}$. Moreover, for a generic $\mathcal{Q},[0: 0: 1]$ is an ordinary intersection point of $V\left(K_{3}\right)$ with $V\left(K_{4}\right)$ and $V\left(K_{3}, K_{4}, z_{1}\right)=\{[0: 0: 1]\}$. Hence $z_{1}$ divides $\operatorname{Res}_{y_{1}}\left(K_{3}, K_{4}\right)$ but not $z_{1}^{-1} \operatorname{Res}_{y_{1}}\left(K_{3}, K_{4}\right)$. The set $\mathcal{E}_{\mathcal{Q}}$ of $\left[y_{2}: z_{2}: t_{2}\right] \in V\left(K_{3}\right)$ such that $\left[z_{2}: t_{2}\right] \in V\left(z_{1}^{-1} \operatorname{Res}_{y_{1}}\left(K_{3}, K_{4}\right)\right) \subset \mathbb{P}^{1}$ is finite. Since $V(F) \not \subset V\left(F_{x}, F_{t}\right), \mathcal{E}_{\mathcal{Q}} \cap V\left(F_{x} \circ \psi, F_{t} \circ \psi\right)=\emptyset$ (up to a linear change of variables in $(y, z)$ ). Hence for a generic $\mathcal{Q}, \mathcal{E}_{\mathcal{Q}} \cap V\left(K_{1}\right)=\emptyset$ and so $\operatorname{Res}_{z_{1}}\left(z_{1}^{-1} \operatorname{Res}_{y_{1}}\left(K_{3}, K_{4}\right), \operatorname{Res}_{y_{1}}\left(K_{1}, K_{3}\right)\right) \neq 0$.

Proof of Proposition 8. Let us write $\mathcal{C}_{0}$ for the set of non singular points of $\mathcal{C}$. Due to the preceding Lemma, the set of quadrics $Q$ such that $\Phi_{\mathcal{C}, \mathcal{Q}}$ is not birational is contained in

$$
\mathcal{K}:=\bigcup_{E \subset \mathcal{C}_{0}: \# E<\infty} \bigcap_{m \in \mathcal{C}_{0} \backslash E} \mathcal{K}_{m}
$$

with $\operatorname{deg} \mathcal{K}_{m} \leq N$. Now, due to a standard argument (see for example [8]), we conclude that either $\mathcal{K}=\emptyset$ or $\mathcal{K}$ is contained in $K_{m_{0}}$ for some $m_{0} \in \mathcal{C}_{0}$. In any case $\mathcal{K}$ is contained in a subvariety of the set of quadrics of $\mathbb{P}^{3}$.

\section{REFERENCES}

[1] J. L. Coolidge. A treatise on algebraic plane curves, Dover, Phenix edition (2004).

[2] I. V. Dolgachev. Classical algebraic geometry. A modern view. Cambridge University Press, Cambridge (2012) xii +639 pp.

[3] D. Eisenbud and J. Harris. Intersection Theory in Algebraic Geometry (2011).

[4] G. H. Halphen. Sur une série de courbes analogues aux développées, Journal de mathématiques pures et appliquées, 3e série, tome 2, p. 87-144 (1876).

[5] G. H. Halphen. Mémoire sur les points singuliers des courbes algébriques planes. Académie des Sciences t. XXVI (1889) No 2.

[6] J. Harris, Algebraic geometry. (English summary) A first course. Corrected reprint of the 1992 original. Graduate Texts in Mathematics, 133. Springer-Verlag, New York, 1995. xx+328 pp.

[7] A. Josse. Transformation d'Halphen. (French) [The Halphen transform] Comm. Algebra 23 (1995), no. 12, 4343-4364.

[8] A. Josse, F. Pène. Degree and class of caustics by reflection for a generic source. C. R. Acad. Sci. Math. 351 (2013) 295-297.

[9] R. Piene, Polar classes of singular varieties, Ann. Sci. École Norm. Sup. (4), 11 no. 2 (1978), 247-274.

[10] R. Piene, Cuspidal projections of space curves, Math. Ann. 256 (1981), no. 1, 95-119.

[11] C. T. C. Wall. Singular Points of Plane Curves. Cambridge University Press. 2004. 
Université de Brest, Laboratoire de Mathématiques de Bretagne Atlantique, UmR CNRS 6205, 29238 Brest cedex, France

E-mail address: alfrederic.josse@univ-brest.fr

Université de Brest and Institut Universitaire de France, Laboratoire de Mathématiques de Bretagne Atlantique, UMR CNRS 6205, 29238 Brest cedex, France

E-mail address: francoise.pene@univ-brest.fr 